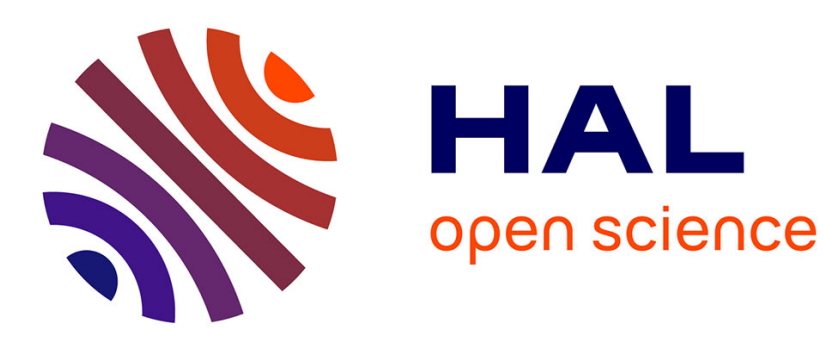

\title{
Generalized formulations of Maxwell's equations for numerical Vlasov-Maxwell simulations
}

\author{
Régine Barthelmé, Patrick Ciarlet, Eric Sonnendrücker
}

\section{To cite this version:}

Régine Barthelmé, Patrick Ciarlet, Eric Sonnendrücker. Generalized formulations of Maxwell's equations for numerical Vlasov-Maxwell simulations. [Research Report] RR-5850, INRIA. 2006, pp.26. inria-00070176

\section{HAL Id: inria-00070176 \\ https://hal.inria.fr/inria-00070176}

Submitted on 19 May 2006

HAL is a multi-disciplinary open access archive for the deposit and dissemination of scientific research documents, whether they are published or not. The documents may come from teaching and research institutions in France or abroad, or from public or private research centers.
L'archive ouverte pluridisciplinaire $\mathbf{H A L}$, est destinée au dépôt et à la diffusion de documents scientifiques de niveau recherche, publiés ou non, émanant des établissements d'enseignement et de recherche français ou étrangers, des laboratoires publics ou privés. 
INSTITUT NATIONAL DE RECHERCHE EN INFORMATIQUE ET EN AUTOMATIQUE

\title{
Generalized formulations of Maxwell's equations for numerical Vlasov-Maxwell simulations
}

\author{
Régine Barthelmé — Patrick Ciarlet — Eric Sonnendrücker
}

\section{$\mathbf{N}^{\circ} \mathbf{5 8 5 0}$}

Février 2006

Thème NUM

\section{apport}

de recherche 



\title{
RINRIA
}

\section{Generalized formulations of Maxwell's equations for numerical Vlasov-Maxwell simulations}

\author{
Régine Barthelm团, Patrick Ciarlet团, Eric Sonnendrücker* \\ Thème NUM - Systèmes numériques \\ Projet Calvi \\ Rapport de recherche $n^{\circ} 5850$ - Février 2006 -26 pages
}

\begin{abstract}
When solving numerically approximations of the Vlasov-Maxwell equations, the source terms in Maxwell's equations coming from the numerical solution of the Vlasov equation do generally not satisfy the continuity equation which is necessary for Maxwell's equations to be well-posed. Hence it is necessary to introduce generalized Maxwell's equations which are still well-posed when there are errors in the sources. Different such formulations have been introduced previously. The aim of this paper is to perform their mathematical analysis and verify the existence and uniqueness of the solution.
\end{abstract}

Key-words: Maxwell; Vlasov-Maxwell; existence; uniqueness.

* IRMA, 7, rue René Descartes, F-67084 Strasbourg cedex et INRIA Lorraine - projet Calvi

$\dagger$ ENSTA/UMA 32, boulevard Victor 75739 Paris Cedex 15 


\section{Formulations généralisées des équations de Maxwell pour les simulations numériques de Vlasov-Maxwell}

Résumé : Lors de la résolution numérique des équations de Vlasov-Maxwell, les termes sources des équations de Maxwell sont calculées à partir d'une solution approchée de l'équation de Vlasov et ne vérifient donc pas en général l'équation de conservation de la charge nécessaire pour que les équations de Maxwell soient bien posées. Il est donc nécessaire d'introduire une formulation généralisée des équations de Maxwell pour garantir l'existence d'une solution pour des termes sources indépendants. Différentes formulations de ce type ont été utilisées dans la littérature. L'objectif de ce rapport est de les analyser et de montrer l'existence et l'unicité de la solution dans différents cas.

Mots-clés : Maxwell; Vlasov-Maxwell; existence; unicité. 


\section{Introduction}

The numerical solution of the coupled Vlasov-Maxwell equations plays a major role in several applications involving interacting charged particles like in plasma physics or in beam physics. The non-relativistic Vlasov-Maxwell system reads

$$
\begin{aligned}
\frac{\partial f}{\partial t}+\mathbf{v} \cdot \nabla_{x} f++\frac{q}{m}(\mathbf{E}+\mathbf{v} \times \mathbf{B}) \cdot \nabla_{v} f & =0, \\
\frac{\partial \mathbf{E}}{\partial t}-c^{2} \operatorname{curl} \mathbf{B} & =-\frac{\mathbf{J}}{\epsilon_{0}}, \\
\frac{\partial \mathbf{B}}{\partial t}+\operatorname{curl} \mathbf{E} & =0, \\
\operatorname{div} \mathbf{E} & =\frac{\rho}{\epsilon_{0}}, \\
\operatorname{div} \mathbf{B} & =0,
\end{aligned}
$$

with $\rho=\int f d \mathbf{v}$ and $\mathbf{J}=\int f \mathbf{v} d \mathbf{v}$. The function $f \equiv f(\mathbf{x}, \mathbf{v}, t)$ represents the particle density in phase space and $\mathbf{E} \equiv \mathbf{E}(\mathbf{x}, t), \mathbf{B} \equiv \mathbf{B}(\mathbf{x}, t)$ are the electric and magnetic fields. A fundamental property of Maxwell's equations is that they are well-posed only for charge and current densities $\rho$ and $\mathbf{J}$ that satisfy the so-called continuity equation

$$
\frac{\partial \rho}{\partial t}+\operatorname{div} \mathbf{J}=0 .
$$

If this relation does not hold, one can verify easily that there is no solution to Maxwell's equations. When the sources of Maxwell's equations are computed by numerically solving the Vlasov equation, they do not in general verify a discrete equivalent to the continuity equation compatible with the discrete form of Maxwell's equations. To address this problem, methods for deriving sources verifying a discrete continuity equation have been introduced, see for example [14. Or, one can choose to perform a correction on the computed electric field so that it remains physically correct. In practice, the latter approach is usually preferred. All those correction methods have been cast into a generic framework [12, 13]. The aim of this paper is to develop a rigorous mathematical theory for these generalized formulations of Maxwell's equations.

The paper is organized as follows. First we recall the different correction types and introduce the generalized Maxwell's equations. After that, we successively prove the existence in an adequate setting of a solution for each formulation.

\section{The different corrections methods}

\subsection{Helmholtz decomposition of a field}

Let us first recall the Helmholtz decomposition of a vector function in $L^{2}(\Omega)^{3}$ into the sum of a transverse (i.e. divergence free) and a longitudinal (i.e. curl free) part. Let $\Omega$ be a bounded open set of $\mathbb{R}^{3}$, with a Lipschitz continuous boundary $\Gamma$.

$\mathrm{RR} \mathrm{n}^{\circ} 5850$ 
For example, the following orthogonal decompositions in $L^{2}(\Omega)^{3}$ can be found in $[8]$ :

$$
\begin{aligned}
L^{2}(\Omega)^{3} & =\underbrace{\nabla H^{1}(\Omega) \oplus \mathbb{H}_{1}(\overbrace{(\Omega)}^{H_{0}(\operatorname{div} 0, \Omega)} \oplus \operatorname{curl} H_{0}(\operatorname{curl}, \Omega)}_{H(\operatorname{curl} 0, \Omega)}, \\
L^{2}(\Omega)^{3} & =\underbrace{\nabla H_{0}^{1}(\Omega) \oplus \mathbb{H}_{2}(\Omega)}_{H_{0}(\operatorname{curl} 0, \Omega)} \oplus \operatorname{curl} H(\operatorname{curl}, \Omega, \Omega)
\end{aligned}
$$

where

$$
\begin{aligned}
& \mathbb{H}_{1}(\Omega)=\left\{\mathbf{u} \in L^{2}(\Omega)^{3} \mid \operatorname{curl} \mathbf{u}=0, \operatorname{div} \mathbf{u}=0, \mathbf{u} \cdot \mathbf{n}_{\left.\right|_{\Gamma}}=0\right\} \\
& \mathbb{H}_{2}(\Omega)=\left\{\mathbf{u} \in L^{2}(\Omega)^{3} \mid \operatorname{curl} \mathbf{u}=0, \operatorname{div} \mathbf{u}=0, \mathbf{u} \times \mathbf{n}_{\left.\right|_{\Gamma}}=0\right\}
\end{aligned}
$$

are finite dimensional vector spaces. The writing $H(\operatorname{curl} 0, \Omega)($ respectively $H(\operatorname{div} 0, \Omega)$ ) means that curl-free (resp. div-free) elements are considered, whereas $H_{0}(\operatorname{curl}, \Omega$ ) (respectively $H_{0}(\operatorname{div}, \Omega)$ ) stand for elements with $L^{2}$ curl (resp. $L^{2}$ div), and such that the tangential (resp. normal) trace on the boundary vanishes (see the next Section for details.)

To summarize, any function in $L^{2}(\Omega)^{3}$ can be decomposed into the sum of a transverse (i.e. divergence free) and longitudinal (i.e. curl free) part. Maxwell's equations can be decomposed in the spaces $\nabla H_{0}^{1}(\Omega)$ and $H(\operatorname{div} 0, \Omega)$ as follows:

$$
\left\{\begin{array} { l } 
{ \partial _ { t } \mathbf { E } _ { L } = - \mathbf { J } _ { L } / \epsilon _ { 0 } } \\
{ \operatorname { d i v } \mathbf { E } _ { L } = \rho / \epsilon _ { 0 } }
\end{array} \quad \left\{\begin{array}{l}
\partial_{t} \mathbf{E}_{T}-c^{2} \operatorname{curl} \mathbf{B}=-\mathbf{J}_{T} / \epsilon_{0} \\
\partial_{t} \mathbf{B}+\operatorname{curl} \mathbf{E}_{T}=0 \\
\operatorname{div} \mathbf{E}_{T}=0 \\
\operatorname{div} \mathbf{B}=0
\end{array}\right.\right.
$$

where $\mathbf{E}=\mathbf{E}_{L}+\mathbf{E}_{T}, \mathbf{J}=\mathbf{J}_{L}+\mathbf{J}_{T}$ with $\mathbf{E}_{L}, \mathbf{J}_{L} \in \nabla H_{0}^{1}(\Omega)$ and $\mathbf{E}_{T}, \mathbf{J}_{T} \in H(\operatorname{div} 0, \Omega)$.

Assume that the continuity equation (1) does not hold. On the one hand, this equation depends only on the longitudinal current density $\mathbf{J}_{L}$ (because $\operatorname{div} \mathbf{J}_{T}=0$ )

$$
\frac{\partial \rho}{\partial t}+\operatorname{div} \mathbf{J}_{L}=0
$$

On the other hand, we notice that the error on the electric field $\mathbf{E}$ that violates the electric Gauss law is localized on the longitudinal part $\mathbf{E}_{L}$ of the field. Consequently, the correction methods will act only on $\mathbf{E}_{L}$. 


\subsection{The Boris correction}

The Boris correction dates back to 1970 [3], [5]. It consists in correcting at each time step the electric field computed with Ampere's law by a longitudinal field deriving from a potential with is computed so that the corrected field exactly satisfies Gauss' law $\operatorname{div} \mathbf{E}=\rho / \epsilon_{0}$. Hence, the longitudinal part of $\mathbf{E}$ is modified as follows:

$$
\mathbf{E}_{\text {corrected }}=\mathbf{E}-\operatorname{grad} \phi
$$

where $\phi$ is defined by

$$
\operatorname{div} \mathbf{E}_{\text {corrected }}=\frac{\rho}{\epsilon_{0}} \Longleftrightarrow \Delta \phi=\operatorname{div} \mathbf{E}-\frac{\rho}{\epsilon_{0}}
$$

and $\phi_{\left.\right|_{\Gamma}}=0$, where $\Gamma$ is the boundary of the computational domain. The field $\mathbf{E}_{\text {corrected }}$ satisfies the same boundary conditions as $\mathbf{E}$. This method is very efficient and very commonly used in applications. However it requires to compute the solution to a Laplace problem at each time step, which has two major drawbacks: it is non local and propagates information at infinite velocity - which is unphysical - and it does not parallelize as well as the explicit expression giving the fields from Ampere's and Faraday's laws, unless ad hoc algorithms are used.

\subsection{The Marder/Langdon correction}

As an alternative to the method introduced in the previous Section, Marder proposed another type of correction in 1987 [11. His method consists in introducing a pseudo-current in Ampere's equation and amounts to computing:

$$
\mathbf{E}_{\text {corrected }}^{n+1}=\mathbf{E}^{n+1}+\Delta t \operatorname{grad}\left[d\left(\operatorname{div} \mathbf{E}^{n}-\frac{\rho^{n}}{\epsilon_{0}}\right)\right]
$$

where $\mathbf{E}^{n+1}$ is the updated field, using Ampere's law. In the case of a a finite difference discretization on a uniform cartesian mesh the diffusion coefficient $d$ must satisfy

$$
d \leq \frac{1}{2 \Delta t}\left(\frac{\Delta x^{2} \Delta y^{2}}{\Delta x^{2}+\Delta y^{2}}\right)
$$

for the method to be stable for a given time step.

Langdon [10] proposed a variation of the method which takes into account the error on Gauss' law at time step $t_{n+1}$ instead of $t_{n}$ :

$$
\mathbf{E}_{\text {corrected }}^{n+1}=\mathbf{E}^{n+1}+\Delta t \operatorname{grad}\left[d\left(\operatorname{div} \mathbf{E}^{n+1}-\frac{\rho^{n+1}}{\epsilon_{0}}\right)\right] .
$$

He also showed that his method is equivalent to performing just one iteration of a Jacobi solver for the Laplace equation used in Boris' method and consequently that his scheme 
Régine Barthelmé, Patrick Ciarlet , Eric Sonnendrücker

can be iterated and converges asymptotically towards Boris' when the iteration number is increased.

More recently, Blaise et al 4 proposed to replace the Jacobi solver by a Gauss-Seidel one. Indeed, it is well-known that the initial convergence rate (over the first iterations) improves dramatically, when one uses a Gauss-Seidel solver. The apparent drawback is that the Jacobi scheme is fully parallel, whereas Gauss-Seidel's is not. Actually, this is not a difficulty, since one recovers a completely parallel algorithm, by renumbering the nodes, using for instance a Red/Black ordering on a structured mesh, as demonstrated in [4].

\subsection{The generalized formulation of Maxwell's equations}

Instead of correcting the longitudinal electric field a posteriori like in the Boris method, Assous et al [2] introduced a mixed formulation of Maxwell's equations with a Lagrange multiplier, that links the Ampere equation to Gauss' law. This solution of Maxwell's equations obtained with this mixed formulation is identical to what is obtained using the Boris correction.

It was then noticed in [12, 13] that Marder's formulation could also be expressed in a modified version of Maxwell's equations, and that the modified Maxwell's equations could be further generalized to include a third kind of natural correction method: after the elliptic correction of Boris, the parabolic correction of Marder a hyperbolic correction could also be included.

Accordingly, the final generalized formulation of Maxwell's equation reads

$$
\begin{aligned}
\frac{\partial \mathbf{E}}{\partial t}-c^{2} \operatorname{curl} \mathbf{B}+c^{2} \operatorname{grad} \phi & =-\frac{\mathbf{J}}{\epsilon_{0}} \\
\frac{\partial \mathbf{B}}{\partial t}+\operatorname{curl} \mathbf{E} & =0 \\
g(\phi)+\operatorname{div} \mathbf{E} & =\frac{\rho}{\epsilon_{0}} \\
\operatorname{div} \mathbf{B} & =0
\end{aligned}
$$

where $g$ is a linear differential operator. It is straightforward to see that the corrector $\phi$ is solution to:

$$
\frac{\partial g(\phi)}{\partial t}-c^{2} \Delta \phi=\frac{1}{\epsilon_{0}}\left(\frac{\partial \rho}{\partial t}+\operatorname{div} \mathbf{J}\right) .
$$

In particular $\phi$ vanishes as soon as the continuity equation is satisfied, assuming appropriate boundary conditions have been chosen. Hence this generalized formulation of Maxwell's equations is equivalent to the original Maxwell's equations in the physical cases but more robust to (numerical) perturbation of the sources. We shall see indeed that this formulation is well-posed independently of a relation between $\rho$ and $\mathbf{J}$.

When $g(\phi)=0$ and $g(\phi)=\phi / d$, the generalized formulation corresponds respectively to the Boris and the Marder/Langdon corrections. In these cases $\phi$ must satisfy vanishing Dirichlet boundary conditions. 
In order to get a purely hyperbolic formulation we choose $g(\phi)=\partial_{t} \phi / \chi^{2}$. The corrector $\phi$ then verifies the following wave equation:

$$
\frac{\partial^{2} \phi}{\partial t^{2}}-(c \chi)^{2} \Delta \phi=\frac{\chi^{2}}{\epsilon_{0}}\left(\frac{\partial \rho}{\partial t}+\operatorname{div} \mathbf{J}\right) .
$$

In order to allow the error transported by the corrector $\phi$ to leave the domain $\Omega$, absorbing boundary conditions must be chosen for $\phi$. These can be for example at order 1:

$$
\frac{\partial \phi}{\partial t}+\left.c \frac{\partial \phi}{\partial \mathbf{n}}\right|_{\Gamma}=0
$$

Remark 1 The numerical implementation of the latter correction method, called hyperbolic correction, is delicate as a good absorption of the outgoing wave is necessary in order to avoid long time amplification of the error. First order absorbing boundary conditions might not be sufficient, and more precise methods like Perfectly Matched Layers (PML) should be preferred.

\section{Notations}

Let $\Omega$ be a bounded open polyhedral set of $\mathbb{R}^{3}$ with a Lipschitz continuous boundary $\Gamma$. We denote by $\mathbf{n}$ the external unit normal defined at almost every point $\Gamma$ of $\Omega$.

The scalar products in $L^{2}(\Omega)^{3}$ and $H^{1}(\Omega)$ are respectively denoted by $(\cdot, \cdot)_{0}$ and $(\cdot, \cdot)_{1}$. Given a Banach space $Q$, we shall denote the duality product in $Q^{\prime} \times Q$ by $\langle., .\rangle_{Q}$.

We recall the definition of the following spaces:

$$
\begin{gathered}
H(\operatorname{div}, \Omega)=\left\{\mathbf{f} \in L^{2}(\Omega)^{3} \mid \operatorname{div} \mathbf{f} \in L^{2}(\Omega)\right\}, \\
H(\operatorname{curl}, \Omega)=\left\{\mathbf{f} \in L^{2}(\Omega)^{3} \mid \operatorname{curl} \mathbf{f} \in L^{2}(\Omega)^{3}\right\},
\end{gathered}
$$

which are Hilbert spaces respectively for the norms:

$$
\begin{aligned}
\|\mathbf{f}\|_{0, \operatorname{div}} & =\left(\int_{\Omega}\left(|\mathbf{f}|^{2}+c^{2}(\operatorname{div} \mathbf{f})^{2}\right) d \mathbf{x}\right)^{1 / 2} \\
\|\mathbf{f}\|_{0, \text { curl }} & =\left(\int_{\Omega}\left(|\mathbf{f}|^{2}+c^{2}|\operatorname{curl} \mathbf{f}|^{2}\right) d \mathbf{x}\right)^{1 / 2} .
\end{aligned}
$$

The mappings $\gamma_{n}$ (normal trace) and $\gamma_{\tau}$ (tangential trace) are respectively defined and continuous on the first and second of these spaces (see [9]):

$$
\begin{gathered}
\gamma_{n}: H(\operatorname{div}, \Omega) \rightarrow H^{-1 / 2}(\Gamma), \mathbf{f} \mapsto \mathbf{f} \cdot \mathbf{n}_{\left.\right|_{\Gamma}}, \\
\gamma_{\tau}: H(\operatorname{curl}, \Omega) \rightarrow H^{-1 / 2}(\Gamma)^{3}, \mathbf{f} \mapsto \mathbf{f} \times \mathbf{n}_{\left.\right|_{\Gamma}}
\end{gathered}
$$

$\mathrm{RR} \mathrm{n}^{\circ} 5850$ 
Hence we can define the following closed subspaces:

$$
\begin{aligned}
H_{0}(\operatorname{div}, \Omega) & =\left\{\mathbf{f} \in H(\operatorname{div}, \Omega) \mid \mathbf{f} \cdot \mathbf{n}_{\left.\right|_{\Gamma}}=0\right\}, \\
H_{0}(\operatorname{curl}, \Omega) & =\left\{\mathbf{f} \in H(\operatorname{curl}, \Omega) \mid \mathbf{f} \times \mathbf{n}_{\left.\right|_{\Gamma}}=0\right\} .
\end{aligned}
$$

We shall denote by

$$
X=H(\operatorname{curl}, \Omega) \cap H(\operatorname{div}, \Omega)
$$

the Hilbert space endowed with the norm

$$
\|\mathbf{f}\|_{X}=\left(\int_{\Omega}\left(|\mathbf{f}|^{2}+c^{2}|\operatorname{curl} \mathbf{f}|^{2}+c^{2}(\operatorname{div} \mathbf{f})^{2} d \mathbf{x}\right)^{1 / 2}\right.
$$

and $X_{0}$ its closed subspace

$$
X_{0}=H_{0}(\operatorname{curl}, \Omega) \cap H(\operatorname{div}, \Omega) .
$$

For a linear space $V \subset H(\operatorname{curl}, \Omega)$, we shall denote by

$$
V(\operatorname{curl})=\left\{\mathbf{f} \in V \mid \operatorname{curl} \operatorname{curl} \mathbf{f} \in L^{2}(\Omega)^{3}\right\} .
$$

Let us also recall a couple of results we shall use later on. Let $H$ and $M$ be two Hilbert spaces. We consider the continuous bilinear form

$$
b: H \times M \rightarrow \mathbb{R},
$$

and the variational problem: Given $\chi \in M^{\prime}$, find $u \in H$ such that

$$
b(u, \mu)=\langle\chi, \mu\rangle \quad \forall \mu \in M .
$$

The bilinear form $b$ is continuous on $H \times M$, hence it defines a bounded linear operator $\mathcal{B} \in \mathcal{L}\left(H ; M^{\prime}\right)$ by

$$
\langle\mathcal{B} v, \mu\rangle=b(v, \mu) \quad \forall v \in H, \quad \forall \mu \in M .
$$

We denote by $\mathcal{B}^{\prime} \in \mathcal{L}\left(M ; H^{\prime}\right)$ the dual operator of $\mathcal{B}$, which is defined by

$$
\left\langle v, \mathcal{B}^{\prime} \mu\right\rangle=\langle\mathcal{B} v, \mu\rangle=b(v, \mu) \quad \forall v \in H, \quad \forall \mu \in M .
$$

The previous variational problem is then equivalent to Find $u \in H$ such that

$$
\mathcal{B} u=\chi \text { in } M^{\prime} .
$$

Set $V=\operatorname{Ker}(\mathcal{B})$, which is a closed linear subspace of $H$, and consider its polar set $V^{\circ}=\left\{g \in H^{\prime} ;\langle g, v>=0 \forall v \in V\}\right.$.

Lemma 1 (Babuska-Brezzi) The following properties are equivalent:

INRIA 
(i) there exists $\beta>0$ such that

$$
\inf _{\mu \in M} \sup _{v \in H} \frac{b(v, \mu)}{\|v\|_{X}\|\mu\|_{M}} \geq \beta
$$

(ii) the operator $\mathcal{B}^{\prime}$ is an isomorphism of $M$ on $V^{\circ}$ and

$$
\left\|\mathcal{B}^{\prime} \mu\right\|_{H^{\prime}} \geq \beta\|\mu\|_{M} \forall \mu \in M
$$

(iii) the operator $\mathcal{B}$ is an isomorphism of $V^{\perp}$ on $M^{\prime}$ and

$$
\|\mathcal{B} v\|_{M^{\prime}} \geq \beta\|v\|_{H} \forall v \in V^{\perp} .
$$

Proof. See the book by Girault and Raviart [9], chap.I, §4.

Definition 1 Let $H$ be a Hilbert space and $A: D(A) \subset H \rightarrow H$ a linear operator.

1) $A$ is monotone if $(A v, v)_{H} \geq 0 \forall v \in D(A)$,

2) $A$ is maximal monotone if moreover there exists $\lambda>0$ such that $I+\lambda A: D(A) \rightarrow H$ is onto.

Theorem 1 (Hille-Yosida) Let $T>0$ and $A$ a maximal monotone operator in $H$. Then for all $u_{0} \in D(A)$ and all $f \in C^{1}([0, T] ; H)$, there exists a unique function u verifying

$$
\left\{\begin{array}{l}
u \in C^{0}([0, T] ; D(A)) \cap C^{1}([0, T] ; H), \\
\frac{d u}{d t}+A u=f \text { on }[0, T], \\
u(0)=u_{0} .
\end{array}\right.
$$

Proof. The proof is given for example in the book of Brézis [6], chap.VII.

Remark 2 Let $\lambda \in \mathbb{R}$. The problem

$$
\left\{\begin{array}{l}
\frac{d u}{d t}+A u+\lambda u=f \text { on }[0, T], \\
u(0)=u_{0} .
\end{array}\right.
$$

can be transformed into (2) with right hand side $e^{\lambda t} f(t)$, by setting $v(t)=e^{\lambda t} u(t)$. Therefore, it is equivalent to prove that $A$ or $A+\lambda I$, for $\lambda \in \mathbb{R}$, is maximal monotone, in order to apply the Hille-Yosida Theorem.

Remark 3 Let $V$ be a dense Hilbert subspace of $H$, and let $a(.,$.$) be a continuous bilinear$ form on $V \times V$. Then the form a defines a bounded linear operator $A \in \mathcal{L}\left(V, V^{\prime}\right)$. In the sequel, we shall also denote by $A$ the linear operator associated to a and defined by

$$
D(A)=\{u \in V ; v \mapsto a(u, v) \text { is continuous on } V \text { for the norm of } H\}
$$

and, for all $u \in D(A),\langle A u, v\rangle=a(u, v) \forall v \in V$. By construction, Au can be extended, thanks to the density of $V$ in $H$, to a continuous linear form on $H$, still denoted by $A u \in H^{\prime}$.

$\mathrm{RR} \mathrm{n}^{\circ} 5850$ 


\section{Well-posedness for the elliptic correction}

We want to solve the following problem (E1): Find E, B, $p$ defined on $\Omega \times[0, T]$ with values in $\mathbb{R}^{3}$ for $\mathbf{E}$ and $\mathbf{B}$, and $\mathbb{R}$ for $p$, verifying the equations

$$
\left\{\begin{aligned}
\frac{\partial \mathbf{E}}{\partial t}-c^{2} \operatorname{curl} \mathbf{B}+c^{2} \nabla p & =-\frac{1}{\epsilon_{0}} \mathbf{J} \\
\frac{\partial \mathbf{B}}{\partial t}+\operatorname{curl} \mathbf{E} & =0 \\
\operatorname{div} \mathbf{E} & =\frac{1}{\epsilon_{0}} \rho,
\end{aligned}\right.
$$

with boundary conditions

$$
\mathbf{E} \times \mathbf{n}=0 \text { on } \Gamma, p=0 \text { on } \Gamma,
$$

and initial conditions

$$
\mathbf{E}(., 0)=\mathbf{E}_{0}, \mathbf{B}(., 0)=\mathbf{B}_{0} \text { and } p(., 0)=0,
$$

satisfying

$$
\begin{gathered}
\operatorname{div} \mathbf{E}_{0}=\rho(., 0) \text { on } \Omega, \operatorname{div} \mathbf{B}_{0}=0 \text { on } \Omega, \\
\mathbf{E}_{0} \times \mathbf{n}=0 \text { on } \Gamma, \mathbf{B}_{0} \cdot \mathbf{n}=0 \text { on } \Gamma .
\end{gathered}
$$

\subsection{The second order uncoupled equations}

The equations for $\mathbf{E}$ and $\mathbf{B}$ can be decoupled by going to the second order in time.

Proposition 1 Problem (E1) is formally equivalent to the following problem (E2): Find E, B, $p$ solutions to:

$$
\left\{\begin{aligned}
\frac{\partial^{2} \mathbf{E}}{\partial t^{2}}+c^{2} \operatorname{curl} \operatorname{curl} \mathbf{E}+c^{2} \nabla \frac{\partial p}{\partial t} & =-\frac{1}{\epsilon_{0}} \frac{\partial \mathbf{J}}{\partial t}, \\
\frac{\partial \mathbf{B}}{\partial t}+\operatorname{curl} \mathbf{E} & =0 \\
\operatorname{div} \mathbf{E} & =\frac{1}{\epsilon_{0}} \rho,
\end{aligned}\right.
$$

with boundary conditions (4) and initial conditions (5) and

$$
\frac{\partial \mathbf{E}}{\partial t}(., 0)=\mathbf{E}_{1}, \text { with } \mathbf{E}_{1}=\operatorname{curl} \mathbf{B}_{0}-\frac{1}{\epsilon_{0}} \mathbf{J}(., 0) .
$$

Proof. If $(\mathbf{E}, \mathbf{B}, p)$ is a sufficiently smooth solution to problem (E1), then, taking the time derivative of the first equation and replacing the term $\partial_{t} \mathbf{B}$ by - curl $\mathbf{E}$, we obtain the first equation of (6). The initial condition (7) is obtained by considering the first equation of (3) at $t=0$ and (5). The rest is identical. 
Reciprocally, let $(\mathbf{E}, \mathbf{B}, p)$ be a solution to problem (E2). Consider

$$
\mathbf{U}=\frac{\partial \mathbf{E}}{\partial t}-c^{2} \operatorname{curl} \mathbf{B}+c^{2} \nabla p+\frac{1}{\epsilon_{0}} \mathbf{J} .
$$

Then:

$$
\frac{\partial \mathbf{U}}{\partial t}=\frac{\partial^{2} \mathbf{E}}{\partial t^{2}}-c^{2} \operatorname{curl} \frac{\partial \mathbf{B}}{\partial t}+c^{2} \nabla \frac{\partial p}{\partial t}+\frac{1}{\epsilon_{0}} \frac{\partial \mathbf{J}}{\partial t}=-c^{2} \operatorname{curl}\left(\operatorname{curl} \mathbf{E}+\frac{\partial \mathbf{B}}{\partial t}\right)=0 .
$$

As $\mathbf{U}(., 0)=0$ according to (7) and (5), if $\mathbf{U}$ is sufficiently smooth in time it implies that $\mathbf{U}=0$, whence the first equation of (3).

\subsection{Variational formulation in $\mathrm{E}$}

From now on, we assume that $\mathbf{J} \in H(\operatorname{div}, \Omega)$, hence in particular $\operatorname{div} \mathbf{J} \in L^{2}(\Omega)$, and $\rho \in L^{2}(\Omega)$, for all $t>0$. For the proof of the final Theorem, we need to introduce $\psi \in H_{0}^{1}(\Omega)$ the unique solution to $\Delta \psi=\operatorname{div} \mathbf{J}$. Then $\widetilde{\mathbf{J}}=\mathbf{J}-\nabla \psi$ automatically verifies $\operatorname{div} \widetilde{\mathbf{J}}=0$.

Proposition 2 Problem (E2) is equivalent to problem (E3), which consists in the mixed variational formulation in $\mathbf{E}$ and $P=-\partial_{t} \psi / \epsilon_{0}-c^{2} \partial_{t} p:$ Find $(\mathbf{E}, P) \in X_{0} \times L^{2}(\Omega)$ such that:

$$
\left\{\begin{array}{l}
\left\langle\frac{\partial^{2} \mathbf{E}}{\partial t^{2}}, \mathbf{F}\right\rangle_{X_{0}}+c^{2}(\operatorname{curl} \mathbf{E}, \operatorname{curl} \mathbf{F})_{0}+(P, \operatorname{div} \mathbf{F})_{0}=-\frac{1}{\epsilon_{0}}\left\langle\frac{\partial \tilde{\mathbf{J}}}{\partial t}, \mathbf{F}\right\rangle_{X_{0}}, \quad \forall \mathbf{F} \in X_{0}, \\
(\operatorname{div} \mathbf{E}, q)_{0}=\frac{1}{\epsilon_{0}}(\rho, q)_{0}, \quad \forall q \in L^{2}(\Omega),
\end{array}\right.
$$

plus $\partial_{t} \mathbf{B}+\operatorname{curl} \mathbf{E}=0$, boundary conditions (4) and initial conditions (5) and (7).

Proof. Assume that problem (E2) has a smooth (see the next Remark) solution (E, $p$ ). Then for all $\mathbf{F} \in X_{0}$, we have

$$
\begin{aligned}
-\frac{1}{\epsilon_{0}}\left\langle\frac{\partial \tilde{\mathbf{J}}}{\partial t}, \mathbf{F}\right\rangle_{X_{0}}= & -\frac{1}{\epsilon_{0}}\left\langle\frac{\partial \mathbf{J}}{\partial t}, \mathbf{F}\right\rangle_{X_{0}}+\frac{1}{\epsilon_{0}}\left(\frac{\partial \nabla \psi}{\partial t}, \mathbf{F}\right)_{0} \\
= & \left\langle\frac{\partial^{2} \mathbf{E}}{\partial t^{2}}, \mathbf{F}\right\rangle_{X_{0}}+c^{2}\langle\operatorname{curl} \operatorname{curl} \mathbf{E}, \mathbf{F}\rangle_{X_{0}}+c^{2}\left\langle\nabla \frac{\partial p}{\partial t}, \mathbf{F}\right\rangle_{X_{0}}+\frac{1}{\epsilon_{0}}\left(\nabla \frac{\partial \psi}{\partial t}, \mathbf{F}\right)_{0} \\
= & \left\langle\frac{\partial^{2} \mathbf{E}}{\partial t^{2}}, \mathbf{F}\right\rangle_{X_{0}}+c^{2}\langle\operatorname{curl} \operatorname{curl} \mathbf{E}, \mathbf{F}\rangle_{H_{0}(\operatorname{curl}, \Omega)}+c^{2}\left\langle\nabla \frac{\partial p}{\partial t}, \mathbf{F}\right\rangle_{H(\operatorname{div}, \Omega)} \\
& +\frac{1}{\epsilon_{0}}\left(\nabla \frac{\partial \psi}{\partial t}, \mathbf{F}\right)_{0} \\
= & \left\langle\frac{\partial^{2} \mathbf{E}}{\partial t^{2}}, \mathbf{F}\right\rangle_{X_{0}}+c^{2}(\operatorname{curl} \mathbf{E}, \operatorname{curl} \mathbf{F})_{0}-\left(c^{2} \frac{\partial p}{\partial t}+\frac{1}{\epsilon_{0}} \frac{\partial \psi}{\partial t}, \operatorname{div} \mathbf{F}\right)_{0} .
\end{aligned}
$$

Set $P=-\partial_{t} \psi / \epsilon_{0}-c^{2} \partial_{t} p$. We then get the mixed variational formulation (8).

The reciprocal assertion is simple: 
- One derives the first equation of (6) in $X_{0}^{\prime}$ provided the solution $(\mathbf{E}, P)$ is smooth enough, if one sets

$$
p(\cdot, t)=-\frac{1}{c^{2}}\left(\int_{0}^{t} P(\cdot, s) d s+\frac{1}{\epsilon_{0}}(\psi(\cdot, t)-\psi(\cdot, 0))\right) \ldots
$$

- The second one is evidently true.

- The third equation holds in $L^{2}(\Omega)$ by construction.

Remark 4 In the previous proof, we evidently assumed that $\partial_{t} \tilde{\mathbf{J}}$ belongs to $X_{0}^{\prime}$.

Now, since $X_{0}$ is a subset of both $H_{0}(\operatorname{curl}, \Omega)$ and of $H(\operatorname{div}, \Omega)$, the converse inclusions hold for the dual spaces. Let us explain here in a few words, what we meant by a smooth solution $(\mathbf{E}, p)$ :

- For $\mathbf{E}$, we assumed that $\operatorname{curl} \mathbf{E} \in L^{2}(\Omega)^{3}$, which implies $\operatorname{curl} \operatorname{curl} \mathbf{E} \in\left(H_{0}(\operatorname{curl}, \Omega)\right)^{\prime}$.

- For $\partial_{t} p$, we assumed that:

(i) $\nabla\left(\partial_{t} p\right) \in(H(\operatorname{div}, \Omega))^{\prime}$;

(ii) $\left\langle\nabla\left(\partial_{t} p\right), \mathbf{F}\right\rangle_{H(\operatorname{div}, \Omega)}=-\left(\partial_{t} p, \operatorname{div} \mathbf{F}\right)_{0}, \forall \mathbf{F} \in H(\operatorname{div}, \Omega)$.

Actually, (ii) corresponds to the boundary condition $p=0$ in (4) in some sense... As soon as $\partial_{t} p$ belongs to $H^{1}(\Omega)$, there holds for, $\mathbf{F} \in H(\operatorname{div}, \Omega)$,

$$
\left\langle\nabla\left(\partial_{t} p\right), \mathbf{F}\right\rangle_{H(\operatorname{div}, \Omega)}=\left(\nabla\left(\partial_{t} p\right), \mathbf{F}\right)_{0}=-\left(\partial_{t} p, \operatorname{div} \mathbf{F}\right)_{0}+\langle\mathbf{F} \cdot \mathbf{n}, p\rangle_{H^{1 / 2}(\Gamma)},
$$

so that $p_{\mid \Gamma}$ vanishes.

The same considerations apply for $(\mathbf{E}, P)$.

Let $b$ be the continuous bilinear form defined on $X_{0} \times L^{2}(\Omega)$ by:

$$
b(u, \lambda)=(\operatorname{div} u, \lambda)_{0} \quad \forall(u, \lambda) \in X_{0} \times L^{2}(\Omega) .
$$

Let $\mathcal{A}: X_{0} \rightarrow X_{0}^{\prime}, \mathcal{B}: X_{0} \rightarrow L^{2}(\Omega)^{\prime}$ and $\mathcal{B}^{\prime}: L^{2}(\Omega) \rightarrow X_{0}^{\prime}$ the linear operators defined by:

$$
\langle\mathcal{A} \mathbf{F}, \mathbf{G}\rangle=c^{2}(\operatorname{curl} \mathbf{F}, \operatorname{curl} \mathbf{G})_{0}, \quad\langle\mathcal{B} \mathbf{F}, q\rangle=(\operatorname{div} \mathbf{F}, q)_{0}, \quad\left\langle\mathcal{B}^{\prime} q, \mathbf{F}\right\rangle=\langle q, \mathcal{B} \mathbf{F}\rangle
$$

for $\mathbf{F}, \mathbf{G} \in X_{0}$ and $q \in L^{2}(\Omega)$. In the sequel, we identify $L^{2}(\Omega)^{\prime}$ with $L^{2}(\Omega)$. It is easy to see that $\mathcal{A}, \mathcal{B}$ and $\mathcal{B}^{\prime}$ are continuous.

The mixed variational formuation (8) can be written equivalently: Find $(\mathbf{E}, P) \in X_{0} \times$ $L^{2}(\Omega)$ such that:

$$
\left\{\begin{aligned}
\frac{\partial^{2} \mathbf{E}}{\partial t^{2}}+\mathcal{A} \mathbf{E}+\mathcal{B}^{\prime} P & =-\frac{1}{\epsilon_{0}} \frac{\partial \tilde{\mathbf{J}}}{\partial t} & \text { in } X_{0}^{\prime} \\
\mathcal{B} \mathbf{E} & =\frac{1}{\epsilon_{0}} \rho & \text { in } L^{2}(\Omega)^{\prime}
\end{aligned}\right.
$$


We shall adapt the proof of Girault and Raviart [9] to show that this time dependent mixed problem has a unique solution. Set

$$
\begin{aligned}
& V=\operatorname{Ker} \mathcal{B}=\left\{v \in X_{0} \mid \operatorname{div} v=0\right\}, \\
& V_{\rho}=\left\{v \in X_{0} \mid \operatorname{div} v=\rho / \epsilon_{0}\right\}, \\
& V^{\circ}=\left\{g \in X_{0}^{\prime} \mid\langle g, v\rangle=0 \quad \forall v \in V\right\}, \\
& V^{\perp}=\left\{u \in X_{0} \mid(u, v)_{X}=0 \quad \forall v \in V\right\} .
\end{aligned}
$$

We note, that given any element $\varphi$ of $H_{0}^{1}(\Omega)$ such that $\Delta \varphi \in L^{2}(\Omega)$, one has $\nabla \varphi \in X_{0}$. In addition, there holds by integration by parts

$$
(\nabla \varphi, v)_{X}=(\nabla \varphi, v)_{0}=0, \forall \varphi \in H_{0}^{1}(\Omega) \text { s.t. } \Delta \varphi \in L^{2}(\Omega), v \in V .
$$

In other words, $\nabla \varphi$ belongs to $V^{\perp}$. This property will be used hereafter.

Lemma 2 The bilinear form $b$ verifies the inf-sup condition in the space $X_{0} \times L^{2}(\Omega)$ :

$$
\inf _{\lambda \in L^{2}(\Omega)} \sup _{v \in X_{0}} \frac{b(v, \lambda)}{\|v\|_{X}\|\lambda\|_{0}} \geq \beta
$$

Proof. We need to prove that there exists $\beta>0$ such that:

$$
\forall \lambda \in L^{2}(\Omega), \quad \sup _{v \in X_{0}} \frac{b(v, \lambda)}{\|v\|_{X}} \geq \beta\|\lambda\|_{0}
$$

Let $\lambda \in L^{2}(\Omega)$. There exists $\xi \in H_{0}^{1}(\Omega)$ such that $\Delta \xi=\lambda$ in $\Omega$. Moreover, $\|\xi\|_{1} \leq C\|\lambda\|_{0}$ with $C>0$ independent of $\lambda$. Then $u=\nabla \xi$ verifies:

$$
\begin{aligned}
& u \in L^{2}(\Omega)^{3}, \\
& \operatorname{div} u=\lambda \in L^{2}(\Omega), \\
& \operatorname{curl} u=0 \in L^{2}(\Omega)^{3}, \\
& u \times \mathbf{n}_{\left.\right|_{\Gamma}}=\nabla \xi \times \mathbf{n}_{\mid \Gamma}=0 \text { as } \xi \in H_{0}^{1}(\Omega) .
\end{aligned}
$$

Hence $u$ belongs to $X_{0}$. On the other hand, $b(u, \lambda)=(\operatorname{div} u, \lambda)_{0}=\|\lambda\|_{0}^{2}$, and

$$
\begin{aligned}
\|u\|_{X}^{2} & =\|u\|_{0}^{2}+\|\operatorname{div} u\|_{0}^{2} \\
& =\|\nabla \xi\|_{0}^{2}+\|\lambda\|_{0}^{2} \\
& \leq\|\xi\|_{1}^{2}+\|\lambda\|_{0}^{2} \\
& \leq\left(1+C^{2}\right)\|\lambda\|_{0}^{2} .
\end{aligned}
$$

Therefore,

$$
\frac{b(u, \lambda)}{\|u\|_{X}}=\|\lambda\|_{0} \frac{\|\lambda\|_{0}}{\|u\|_{X}} \geq\|\lambda\|_{0}\left(1+C^{2}\right)^{-1 / 2}
$$

$\mathrm{RR} \mathrm{n}^{\circ} 5850$ 
and so

$$
\sup _{v \in X_{0}} \frac{b(v, \lambda)}{\|v\|_{X}} \geq \frac{b(u, \lambda)}{\|u\|_{X}} \geq \beta\|\lambda\|_{0}
$$

with $\beta=\left(1+C^{2}\right)^{-1 / 2}$ independent of $\lambda$.

Theorem 2 Assume that the initial data verify

$$
\left(\mathbf{E}_{0}, \mathbf{E}_{1}\right) \in X_{0}(\operatorname{curl}) \times X_{0},
$$

and that the sources $\rho$ and $\mathbf{J}$ satisfy

$$
\mathbf{J} \in \mathcal{C}^{2}([0, T] ; H(\operatorname{div}, \Omega)), \quad \rho \in \mathcal{C}^{2}\left([0, T] ; L^{2}(\Omega)\right), \quad\left(\frac{\partial \rho}{\partial t}+\operatorname{div} \mathbf{J}\right)(., 0)=0 .
$$

Then the mixed variational formulation (8) has a unique solution $(\mathbf{E}, P)$, verifying

$$
\begin{gathered}
\mathbf{E} \in \mathcal{C}^{0}\left([0, T] ; X_{0}(\operatorname{curl})\right) \cap \mathcal{C}^{1}\left([0, T] ; X_{0}\right) \cap \mathcal{C}^{2}([0, T] ; H(\operatorname{div}, \Omega)), \\
P \in \mathcal{C}^{0}\left([0, T] ; L^{2}(\Omega)\right) .
\end{gathered}
$$

Proof. 1. On the one hand, the bilinear form $b$ verifies the inf-sup condition in the space $X_{0} \times L^{2}(\Omega)$. Then, according to Lemma 1, $\mathcal{B}$ is an isomorphism from $V^{\perp}$ onto $L^{2}(\Omega)^{\prime}$. Hence there exists a unique $\mathbf{E}^{\perp} \in V^{\perp}$ such that

$$
\mathcal{B} \mathbf{E}^{\perp}=\rho / \epsilon_{0} .
$$

Moreover, due to inequality (iii) in Lemma 1 and as $\rho \in \mathcal{C}^{2}\left([0, T] ; L^{2}(\Omega)\right)$,

$$
\mathbf{E}^{\perp} \in \mathcal{C}^{2}\left([0, T] ; V^{\perp}\right) .
$$

On the other hand, let $\varphi \in H_{0}^{1}(\Omega)$ be a solution to $\Delta \varphi=\rho / \epsilon_{0}$. According to (10), $\nabla \varphi \in V^{\perp}$. By uniqueness $\left(\mathcal{B}\right.$ is an isomorphism on $\left.V^{\perp}\right), \mathbf{E}^{\perp}=\nabla \varphi$. This implies that

$$
\mathcal{A} \mathbf{E}^{\perp}=0, \quad \text { and }\left(\mathbf{E}^{\perp}, \mathbf{F}\right)_{X}=\left(\mathbf{E}^{\perp}, \mathbf{F}\right)_{0}=0 \text { for all } \mathbf{F} \in V .
$$

2. Denote by $\mathcal{A}_{V}$ the linear operator defined by $\mathcal{A}_{V}: X_{0} \rightarrow V^{\prime}, \mathbf{F} \mapsto \mathcal{A}_{V} \mathbf{F}$, such that

$$
\left\langle\mathcal{A}_{V} \mathbf{F}, \mathbf{G}\right\rangle=c^{2}(\operatorname{curl} \mathbf{F}, \operatorname{curl} \mathbf{G})_{0}, \forall(\mathbf{F}, \mathbf{G}) \in X_{0} \times V .
$$

If $(\mathbf{E}, P)$ is a solution to the mixed problem (9), it implies that $\mathbf{E}$ is a solution to the following problem restricted to $V$ : Find $\mathbf{E} \in V_{\rho}$ such that:

$$
\frac{\partial^{2} \mathbf{E}}{\partial t^{2}}+\mathcal{A}_{V} \mathbf{E}=-\frac{1}{\epsilon_{0}} \frac{\partial \tilde{\mathbf{J}}}{\partial t} \text { in } V^{\prime} .
$$

According to (10), this problem is equivalent to: Find $\mathbf{W}=\mathbf{E}-\mathbf{E}^{\perp} \in V$ such that:

$$
\frac{\partial^{2} \mathbf{W}}{\partial t^{2}}+\mathcal{A}_{V} \mathbf{W}=-\frac{1}{\epsilon_{0}} \frac{\partial \tilde{\mathbf{J}}}{\partial t} \text { in } V^{\prime} .
$$

INRIA 
Setting

$$
u=\left(\begin{array}{c}
\mathbf{W} \\
\partial_{t} \mathbf{W}
\end{array}\right), A=\left(\begin{array}{cc}
0 & -I \\
\mathcal{A}_{V} & 0
\end{array}\right), f=\left(\begin{array}{c}
0 \\
-\partial_{t} \mathbf{J} / \epsilon_{0}
\end{array}\right), u_{0}=\left(\begin{array}{c}
\mathbf{E}_{0}-\mathbf{E}^{\perp}(., 0) \\
\mathbf{E}_{1}-\partial_{t} \mathbf{E}^{\perp}(., 0)
\end{array}\right),
$$

this problem becomes

$$
\frac{d}{d t} u+A u=f, u(0)=u_{0}
$$

Introduce

$$
\begin{aligned}
H & =V \times H(\operatorname{div} 0, \Omega) \\
D(A) & =\{u \in H \mid A u \in H\}=V(\operatorname{curl}) \times V .
\end{aligned}
$$

Let us admit for the moment the following Lemma.

Lemma 3 The operator $I+A: D(A) \rightarrow H$ is maximal monotone on $H$.

According to Remark 2] we can apply the Hille-Yosida Theorem: assuming that $u_{0} \in$ $D(A)$ and $f \in \mathcal{C}^{1}([0, T], H)$, problem (14) has a unique solution $u \in \mathcal{C}^{0}([0, T], D(A)) \cap$ $\mathcal{C}^{1}([0, T], H)$.

The hypotheses of the Hille-Yosida Theorem correspond to

- $f \in \mathcal{C}^{1}([0, T], H) \Longleftrightarrow \partial_{t} \tilde{\mathbf{J}} \in \mathcal{C}^{1}([0, T], H(\operatorname{div} 0, \Omega))$.

The right side holds when $\mathbf{J} \in \mathcal{C}^{2}([0, T] ; H(\operatorname{div}, \Omega))$ and $\rho \in \mathcal{C}^{2}\left([0, T] ; L^{2}(\Omega)\right)$.

- $u_{0} \in D(A) \Longleftrightarrow \mathbf{E}_{0}-\mathbf{E}^{\perp}(., 0) \in V(\operatorname{curl})$ and $\mathbf{E}_{1}-\partial_{t} \mathbf{E}^{\perp}(., 0) \in V$.

Because of the hypotheses and as curl $\mathbf{E}^{\perp}=0$, one has $\mathbf{E}_{0}-\mathbf{E}^{\perp}(., 0) \in X_{0}$ (curl). Moreover,

$$
\operatorname{div}\left(\mathbf{E}_{0}-\mathbf{E}^{\perp}(., 0)\right)=\operatorname{div} \mathbf{E}_{0}-\rho(., 0) / \epsilon_{0}=0,
$$

hence $\mathbf{E}_{0}-\mathbf{E}^{\perp}(., 0)$ belongs to $V(\operatorname{curl})$. In the same way, $\mathbf{E}_{1}-\partial_{t} \mathbf{E}^{\perp}(., 0) \in X_{0}$, and because of (7):

$$
\begin{aligned}
\operatorname{div}\left(\mathbf{E}_{1}-\frac{\partial \mathbf{E}^{\perp}}{\partial t}(., 0)\right) & =\operatorname{div} \mathbf{E}_{1}-\frac{1}{\epsilon_{0}} \frac{\partial \rho}{\partial t}(., 0) \\
& =\operatorname{div}\left(c^{2} \operatorname{curl} \mathbf{B}_{0}-\mathbf{J}(., 0) / \epsilon_{0}\right)-\frac{1}{\epsilon_{0}} \frac{\partial \rho}{\partial t}(., 0) \\
& =-\frac{1}{\epsilon_{0}}\left(\operatorname{div} \mathbf{J}+\frac{\partial \rho}{\partial t}\right)(., 0)=0 .
\end{aligned}
$$

Hence $\mathbf{E}_{1}-\partial_{t} \mathbf{E}^{\perp}(., 0)$ belongs to $V$.

Therefore, the hypotheses of the Hille-Yosida Theorem are all verified, and we get existence and uniqueness of

$$
u \in \mathcal{C}^{0}([0, T], D(A)) \cap \mathcal{C}^{1}([0, T], H),
$$


which is equivalent to

$$
\mathbf{W} \in \mathcal{C}^{0}([0, T], V(\operatorname{curl})) \cap \mathcal{C}^{1}([0, T], V) \cap \mathcal{C}^{2}([0, T], H(\operatorname{div} 0, \Omega)),
$$

unique solution to (13). Since $X_{0}=V \oplus V^{\perp}$, we have existence and uniqueness of the solution $\mathbf{E}$ to (9), according to the splitting

$$
\mathbf{E}=\mathbf{W}+\mathbf{E}^{\perp} \in \mathcal{C}^{0}\left([0, T], X_{0}(\operatorname{curl})\right) \cap \mathcal{C}^{1}\left([0, T], X_{0}\right) \cap \mathcal{C}^{2}([0, T], H(\operatorname{div}, \Omega)) .
$$

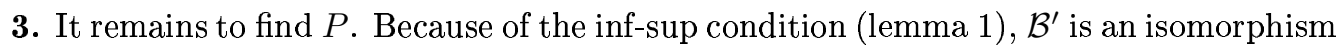
from $L^{2}(\Omega)$ on $V^{\circ}$. But

$$
-\frac{1}{\epsilon_{0}} \frac{\partial \tilde{\mathbf{J}}}{\partial t}-\frac{\partial^{2} \mathbf{E}}{\partial t^{2}}-\mathcal{A} \mathbf{E}=\left(-\frac{1}{\epsilon_{0}} \frac{\partial \tilde{\mathbf{J}}}{\partial t}-\frac{\partial^{2} \mathbf{W}}{\partial t^{2}}-\mathcal{A} \mathbf{W}\right)+\left(-\frac{\partial^{2} \mathbf{E}^{\perp}}{\partial t^{2}}-\mathcal{A} \mathbf{E}^{\perp}\right) \in V^{\circ}
$$

Hence there exists a unique $P$ in $L^{2}(\Omega)$ such that

$$
\mathcal{B}^{\prime} P=-\frac{1}{\epsilon_{0}} \frac{\partial \tilde{\mathbf{J}}}{\partial t}-\frac{\partial^{2} \mathbf{E}}{\partial t^{2}}-\mathcal{A} \mathbf{E} \in V^{\circ} .
$$

Using now inequality (ii) of Lemma 1 and the fact that

$$
-\frac{\partial \tilde{\mathbf{J}}}{\partial t}-\frac{\partial^{2} \mathbf{E}}{\partial t^{2}}-\mathcal{A} \mathbf{E} \in \mathcal{C}^{0}\left([0, T], L^{2}(\Omega)^{3}\right)
$$

we obtain that

$$
P \in \mathcal{C}^{0}\left([0, T] ; L^{2}(\Omega)\right)
$$

To complete the proof, we need to prove Lemma 3

Proof. [Proof of Lemma 3] Let us show that $I+A$ is maximal monotone on $H$.

Consider $u \in D(A)$.

$$
\begin{aligned}
((I+A) u, u)_{H} & =\left(u_{1}-u_{2}, u_{1}\right)_{V}+\left(u_{2}+\mathcal{A}_{V} u_{1}, u_{2}\right)_{0, \operatorname{div} 0} \\
& =\int_{\Omega}\left(\left|u_{1}\right|^{2}+c^{2}\left|\operatorname{curl} u_{1}\right|^{2}-u_{1} \cdot u_{2}-c^{2} \operatorname{curl} u_{1} \cdot \operatorname{curl} u_{2}\right. \\
& \left.\quad+\left|u_{2}\right|^{2}+c^{2} \operatorname{curl} u_{1} \cdot \operatorname{curl} u_{2}\right) d \mathbf{x} \\
& =\int_{\Omega}\left(c^{2}\left|\operatorname{curl} u_{1}\right|^{2}+\left|u_{1}\right|^{2}+\left|u_{2}\right|^{2}-u_{2} \cdot u_{1}\right) d \mathbf{x} \geq 0 .
\end{aligned}
$$

Hence the operator $I+A$ is monotone.

Consider now $f \in H$. We look for $u \in D(A)$ such that $(2 I+A) u=f$, that is, we look for $u_{1} \in V(\operatorname{curl})$ and $u_{2} \in V$ such that

$$
\left\{\begin{array}{l}
2 u_{1}-u_{2}=f_{1} \in V \\
2 u_{2}+\mathcal{A}_{V} u_{1}=f_{2} \in H(\operatorname{div} 0, \Omega) .
\end{array}\right.
$$

INRIA 
This system is equivalent to

$$
\left\{\begin{array}{l}
u_{2}=2 u_{1}-f_{1} \\
4 u_{1}+\mathcal{A}_{V} u_{1}=f_{2}+2 f_{1} .
\end{array}\right.
$$

The bilinear form $\left(u_{1}, v_{1}\right) \mapsto\left(4 u_{1}, v_{1}\right)_{0}+c^{2}\left(\operatorname{curl} u_{1}, \operatorname{curl} v_{1}\right)_{0}$ is continuous and coercive on the closed subspace $V$ of $X_{0} . f_{2}+2 f_{1} \in H(\operatorname{div} 0, \Omega)$ defines a linear continuous form on $V$. Due to the Lax-Milgram Theorem, there exists a unique $u_{1} \in V$ such that

$$
\left\langle 4 u_{1}+\mathcal{A}_{V} u_{1}, v_{1}\right\rangle_{V}=\left(f_{2}+2 f_{1}, v_{1}\right) \text { for all } v_{1} \in V .
$$

We prove now that $c^{2} \operatorname{curl} \operatorname{curl} u_{1}=f_{2}+2 f_{1}-4 u_{1}$ in the sense of distributions.

Let $\varphi$ be any function in $\mathcal{D}(\Omega)$. There exists a unique $\lambda \in H_{0}^{1}(\Omega)$ such that $\Delta \lambda=\operatorname{div} \varphi$. Set $\psi=\varphi-\nabla \lambda$ : we verify easily that $(\nabla \lambda, \psi) \in H_{0}(\operatorname{curl}, \Omega) \times V$. One has, by definition of $u_{1}$,

$$
\left\langle c^{2} \operatorname{curl} \operatorname{curl} u_{1}, \psi\right\rangle_{V}=\left\langle\mathcal{A}_{V} u_{1}, \psi\right\rangle_{V}=\left(f_{2}+2 f_{1}-4 u_{1}, \psi\right)_{0} .
$$

Moreover

$$
\left\langle c^{2} \operatorname{curl} \operatorname{curl} u_{1}, \nabla \lambda\right\rangle_{H_{0}(\operatorname{curl}, \Omega)}=0 .
$$

Therefore, $c^{2} \operatorname{curl} \operatorname{curl} u_{1}=f_{2}+2 f_{1}-4 u_{1}$ follows in $\mathcal{D}(\Omega)^{\prime}$ and so $u_{1} \in V(\operatorname{curl})$.

We deduce the existence of $u_{2}=2 u_{1}-f_{1} \in V$. The operator $2 I+A$ is onto on $H$, whence $I+A$ is maximal monotone on $H$.

The initial problem can finally be solved.

Theorem 3 Under hypotheses (11), (12) and

$$
\mathbf{B}_{0} \in H_{0}(\operatorname{div} 0, \Omega) \cap H(\operatorname{curl}, \Omega),
$$

problem (E2) has a unique solution $(\mathbf{E}, \mathbf{B}, p)$ verifying

$$
\begin{aligned}
& \mathbf{E} \in \mathcal{C}^{0}\left([0, T], X_{0}(\operatorname{curl})\right) \cap \mathcal{C}^{1}\left([0, T], X_{0}\right) \cap \mathcal{C}^{2}\left([0, T], L^{2}(\Omega)^{3}\right), \\
& \mathbf{B} \in \mathcal{C}^{1}\left([0, T], H_{0}(\operatorname{div} 0, \Omega) \cap H(\operatorname{curl}, \Omega)\right) \cap \mathcal{C}^{2}\left([0, T], H_{0}(\operatorname{div} 0, \Omega)\right), \\
& p \in \mathcal{C}^{1}\left([0, T], H_{0}^{1}(\Omega)\right) .
\end{aligned}
$$

Proof. Starting from the weak solution $(\mathbf{E}, P)$ obtained from Theorem 2 we build $\mathbf{B}$ and $p$, then we prove that $(\mathbf{E}, \mathbf{B}, p)$ is a strong solution to problem (E2).

Due to (8), $\operatorname{div} \mathbf{E}=\rho / \epsilon_{0}$ in $L^{2}(\Omega)$ for all $t$. The boundary condition $\mathbf{E} \times \mathbf{n}_{\left.\right|_{\Gamma}}=0$ follows from the fact that $\mathbf{E}$ is in the space $X_{0}$. For all $\phi \in \mathcal{D}(\Omega)^{3}$, we have

$$
\left(\frac{\partial^{2} \mathbf{E}}{\partial t^{2}}+c^{2} \operatorname{curl} \operatorname{curl} \mathbf{E}-\nabla P, \phi\right)_{0}=-\frac{1}{\epsilon_{0}}\left(\frac{\partial \tilde{\mathbf{J}}}{\partial t}, \phi\right)_{0},
$$


hence

$$
\nabla P=\frac{1}{\epsilon_{0}} \frac{\partial \tilde{\mathbf{J}}}{\partial t}+\frac{\partial^{2} \mathbf{E}}{\partial t^{2}}+c^{2} \operatorname{curl} \operatorname{curl} \mathbf{E} \text { in } L^{2}(\Omega) .
$$

We deduce that $P \in \mathcal{C}^{0}\left([0, T], H^{1}(\Omega)\right)$. Thus, $P_{\left.\right|_{\Gamma}}$ has a meaning in $L^{2}(\Gamma)$. To prove that it is equal to zero, let us take sufficiently many test functions... Recall that $\Omega$ is a polyhedron, so that its boundary is composed of faces $\left(\Gamma_{k}\right)_{k}$. The union $\cup_{k} \mathcal{D}\left(\Gamma_{k}\right)$ is dense in $L^{2}(\Gamma)$. So, for a given face $\Gamma_{k_{0}}$, let $\lambda \in \mathcal{D}\left(\Gamma_{k_{0}}\right)$. According to Proposition 3.2 of [1], there exists $v \in X_{0}$ such that $v \cdot \mathbf{n}_{\left.\right|_{\Gamma}}=\lambda$. Using this $v$ as a test function in (8), we find that

$$
0=\int_{\Gamma} P v \cdot \mathbf{n} d \Gamma=\int_{\Gamma_{k_{0}}} P \lambda d \Gamma .
$$

In other words, $P_{\left.\right|_{\Gamma}}=0$ and $P \in \mathcal{C}^{0}\left([0, T], H_{0}^{1}(\Omega)\right)$.

On the other hand $\mathbf{J} \in \mathcal{C}^{2}([0, T], H(\operatorname{div}, \Omega))$, whence $\psi \in \mathcal{C}^{2}\left([0, T], H_{0}^{1}(\Omega)\right)$. From the equality

$$
\frac{\partial p}{\partial t}=\frac{1}{c^{2}}\left(-\frac{1}{\epsilon_{0}} \frac{\partial \psi}{\partial t}-P\right)
$$

and from $p(., 0)=0$, we finally get $p \in \mathcal{C}^{1}\left([0, T], H_{0}^{1}(\Omega)\right)$. Let now $\mathbf{B}$ be a solution to

$$
\frac{\partial \mathbf{B}}{\partial t}=-\operatorname{curl} \mathbf{E}, \quad \mathbf{B}(., 0)=\mathbf{B}_{0} .
$$

We know that $\operatorname{curl} \mathbf{E} \in \mathcal{C}^{0}\left([0, T], H_{0}(\operatorname{div} 0, \Omega) \cap H(\operatorname{curl}, \Omega)\right) \cap \mathcal{C}^{1}\left([0, T], H_{0}(\operatorname{div} 0, \Omega)\right)$, since

$$
\operatorname{curl} \mathbf{E} \cdot \mathbf{n}_{\mid \Gamma}=\operatorname{curl}(\mathbf{E} \times \mathbf{n})_{\mid \Gamma}=0 .
$$

As $\mathbf{B}_{0} \in H_{0}(\operatorname{div} 0, \Omega) \cap H(\operatorname{curl}, \Omega)$, we finally get $\mathbf{B} \in \mathcal{C}^{1}\left([0, T], H_{0}(\operatorname{div} 0, \Omega) \cap H(\operatorname{curl}, \Omega)\right) \cap$ $\mathcal{C}^{2}\left([0, T], H_{0}(\operatorname{div} 0, \Omega)\right)$.

\section{Well-posedness for the parabolic correction}

Problem (P1) consists in finding $\mathbf{E}, \mathbf{B}: \Omega \times[0, T] \rightarrow \mathbb{R}^{3}$ and $p: \Omega \times[0, T] \rightarrow \mathbb{R}$ such that

$$
\left\{\begin{array}{l}
\frac{\partial \mathbf{E}}{\partial t}-c^{2} \operatorname{curl} \mathbf{B}+c^{2} \nabla p=-\frac{1}{\epsilon_{0}} \mathbf{J} \\
\frac{\partial \mathbf{B}}{\partial t}+\operatorname{curl} \mathbf{E}=0 \\
p+\operatorname{div} \mathbf{E}=\frac{\rho}{\epsilon_{0}}
\end{array}\right.
$$

along with the initial conditions (5) and the boundary conditions (4).

The main difference with the previous Section (elliptic correction) is that, since one can replace $p$ by $\rho / \epsilon_{0}-\operatorname{div} \mathbf{E}$, there is no need for a mixed (or saddle-point) formulation involving both $\mathbf{E}$ and $p$. Proofs will then be technically simpler here...

INRIA 
To simplify the setting further, we can consider that $\rho=0$. Indeed if $\rho \in \mathcal{C}^{k}\left([0, T], L^{2}(\Omega)\right)$, there exists $\varphi \in \mathcal{C}^{k}\left([0, T], H_{0}^{1}(\Omega)\right)$ such that $\Delta \varphi=\rho / \epsilon_{0}$, i. e. $\nabla \varphi$ roughly plays the role of $\mathbf{E}^{\perp}$ of Subsection 4.2 Setting $\mathbf{W}=\mathbf{E}-\nabla \varphi$ and $\tilde{\mathbf{J}}=\mathbf{J}+\epsilon_{0} \partial_{t}(\nabla \varphi)$, the previous problem can be written with a vanishing source term in the last equation. We thus consider that $\rho=0$ in the sequel.

\subsection{The second order uncoupled equations}

Proposition 3 Problem (P1) is equivalent to problem (P2): Find E, B, $p$ solutions to

$$
\left\{\begin{array}{l}
\frac{\partial^{2} \mathbf{E}}{\partial t^{2}}+c^{2} \operatorname{curl} \operatorname{curl} \mathbf{E}-c^{2} \nabla \operatorname{div} \frac{\partial \mathbf{E}}{\partial t}=-\frac{1}{\epsilon_{0}} \frac{\partial \mathbf{J}}{\partial t} \\
\frac{\partial \mathbf{B}}{\partial t}=-\operatorname{curl} \mathbf{E} \\
p=-\operatorname{div} \mathbf{E}
\end{array}\right.
$$

with boundary conditions (4) and initial conditions (5) and (7).

The proof is omitted, since it is very similar to the proof of Proposition 1

\subsection{Variational formulation in $\mathrm{E}$}

To build a variational formulation, let us assume that there exists a smooth solution $(\mathbf{E}, \mathbf{B}, p)$ to problem (P2). As to the required smoothness, we refer the reader to Remark 4 , with $p$ replaced by $\partial_{t} p=-\partial_{t} \operatorname{div} \mathbf{E}$. Given $\mathbf{F}$ in $X_{0}$, we have

$$
\begin{aligned}
-\frac{1}{\epsilon_{0}}\left\langle\frac{\partial \mathbf{J}}{\partial t}, \mathbf{F}\right\rangle_{X_{0}} & =\left\langle\frac{\partial^{2} \mathbf{E}}{\partial t^{2}}, \mathbf{F}\right\rangle_{X_{0}}+c^{2}\langle\operatorname{curl} \operatorname{curl} \mathbf{E}, \mathbf{F}\rangle_{X_{0}}-c^{2}\left\langle\nabla \operatorname{div} \frac{\partial \mathbf{E}}{\partial t}, \mathbf{F}\right\rangle_{X_{0}} \\
& =\left\langle\frac{\partial^{2} \mathbf{E}}{\partial t^{2}}, \mathbf{F}\right\rangle_{X_{0}}+c^{2}\langle\operatorname{curl} \operatorname{curl} \mathbf{E}, \mathbf{F}\rangle_{H_{0}(\operatorname{curl}, \Omega)^{\prime}}-c^{2}\left\langle\nabla \operatorname{div} \frac{\partial \mathbf{E}}{\partial t}, \mathbf{F}\right\rangle_{H(\operatorname{div}, \Omega)} \\
& =\left\langle\frac{\partial^{2} \mathbf{E}}{\partial t^{2}}, \mathbf{F}\right\rangle_{X_{0}}+c^{2}(\operatorname{curl} \mathbf{E}, \operatorname{curl} \mathbf{F})_{0}+c^{2}\left(\operatorname{div} \frac{\partial \mathbf{E}}{\partial t}, \operatorname{div} \mathbf{F}\right)_{0} .
\end{aligned}
$$

Above, we are looking for $\mathbf{E} \in X_{0}$. This variational formulation can be written equivalently: find $\mathbf{E} \in X_{0}$ such that

$$
\frac{\partial^{2} \mathbf{E}}{\partial t^{2}}+\mathcal{A} \mathbf{E}+\mathcal{B} \frac{\partial \mathbf{E}}{\partial t}=-\frac{1}{\epsilon_{0}} \frac{\partial \mathbf{J}}{\partial t} \text { in } X_{0}^{\prime}
$$

where the linear forms are now defined by

$$
\langle\mathcal{A} \mathbf{F}, \mathbf{G}\rangle=c^{2}(\operatorname{curl} \mathbf{F}, \operatorname{curl} \mathbf{G})_{0}, \quad\langle\mathcal{B} \mathbf{F}, \mathbf{G}\rangle=c^{2}(\operatorname{div} \mathbf{F}, \operatorname{div} \mathbf{G})_{0},
$$

for $\mathbf{F}, \mathbf{G} \in X_{0}$. From problem (17), one can simply rebuild the first equation of (16) in $X_{0}^{\prime}$, provided again $\mathbf{E}$ is smooth enough. 
The boundary condition on $\mathbf{E}$ follows from the definition of the space $X_{0}=\mathrm{H}_{0}(\operatorname{curl}, \Omega) \cap$ $\mathrm{H}(\operatorname{div}, \Omega)$. Finally, the one on $p=-\operatorname{div} \mathbf{E}$ is contained in the variational formulation.

Set $u=\left(\begin{array}{c}\mathbf{E} \\ \partial_{t} \mathbf{E}\end{array}\right), A=\left(\begin{array}{cc}0 & -I \\ \mathcal{A} & \mathcal{B}\end{array}\right), f=\left(\begin{array}{c}0 \\ -\partial_{t} \mathbf{J} / \epsilon_{0}\end{array}\right)$ and $u_{0}=\left(\begin{array}{l}\mathbf{E}_{0} \\ \mathbf{E}_{1}\end{array}\right)$. The problem (17) with initial conditions (5) and (7) then reads

$$
\frac{d u}{d t}+A u=f \text { in } X_{0}^{\prime 2}, \quad u(., 0)=u_{0} .
$$

Set $H=X_{0} \times L^{2}(\Omega)^{3}$ and $D(A)=\left\{u \in X_{0} \times X_{0} ; \mathcal{A} u_{1}+\mathcal{B} u_{2} \in L^{2}(\Omega)^{3}\right\}$.

Lemma 4 The operator $I+A: D(A) \rightarrow H$ is maximal monotone.

Proof. Let $u \in D(A)$. Then

$$
\begin{aligned}
((I+A) u, u)_{H}= & \left(u_{1}-u_{2}, u_{1}\right)_{X_{0}}+\left(u_{2}+\mathcal{A} u_{1}+\mathcal{B} u_{2}, u_{2}\right)_{0} \\
= & \int_{\Omega}\left(\left|u_{1}\right|^{2}+c^{2}\left|\operatorname{curl} u_{1}\right|^{2}+c^{2}\left(\operatorname{div} u_{1}\right)^{2}-u_{1} \cdot u_{2}-c^{2} \operatorname{curl} u_{1} \cdot \operatorname{curl} u_{2}\right. \\
& \left.\quad-c^{2} \operatorname{div} u_{1} \operatorname{div} u_{2}+\left|u_{2}\right|^{2}+c^{2} \operatorname{curl} u_{1} \cdot \operatorname{curl} u_{2}+c^{2}\left(\operatorname{div} u_{2}\right)^{2}\right) d x \\
& =\int_{\Omega}\left(c^{2}\left|\operatorname{curl} u_{1}\right|^{2}+c^{2}\left(\operatorname{div} u_{1}\right)^{2}+c^{2}\left(\operatorname{div} u_{2}\right)^{2}-c^{2} \operatorname{div} u_{2} \operatorname{div} u_{1}\right. \\
& \left.\quad+\left|u_{1}\right|^{2}+\left|u_{2}\right|^{2}-u_{2} \cdot u_{1}\right) d x \\
\geq & 0 .
\end{aligned}
$$

Hence $I+A$ is monotone. Let $f \in H$. We look for $u \in D(A)$ such that $(2 I+A) u=f$, that is

$$
\left\{\begin{array}{l}
2 u_{1}-u_{2}=f_{1} \in X_{0} \\
2 u_{2}+\mathcal{A} u_{1}+\mathcal{B} u_{2}=f_{2} \in L^{2}(\Omega)^{3}
\end{array}\right.
$$

This system is equivalent to

$$
\left\{\begin{array}{l}
u_{1}=\frac{1}{2}\left(u_{2}+f_{1}\right) \\
4 u_{2}+\mathcal{A} u_{2}+2 \mathcal{B} u_{2}=2 f_{2}-\mathcal{A} f_{1} .
\end{array}\right.
$$

As $f_{1} \in X_{0}$ and $f_{2} \in L^{2}(\Omega)^{3}, 2 f_{2}-\mathcal{A} f_{1}$ is a continuous linear form on $X_{0}$. The bilinear form $\left(u_{2}, v_{2}\right) \mapsto<4 u_{2}+\mathcal{A} u_{2}+2 \mathcal{B} u_{2}, v_{2}>_{X_{0}}$ is continuous and coercive on $X_{0}^{2}$. Due to the Lax-Milgram Theorem, there exists $u_{2} \in X_{0}$ such that $(4 I+\mathcal{A}+2 \mathcal{B}) u_{2}=2 f_{2}-\mathcal{A} f_{1}$ in $X_{0}^{\prime}$. Then $u_{1}=\frac{1}{2}\left(u_{2}+f_{1}\right) \in X_{0}$ and $\mathcal{A} u_{1}+\mathcal{B} u_{2}=f_{2}-2 u_{2} \in L^{2}(\Omega)^{3}$. Hence $2 I+A$ is onto and the operator $I+A$ is maximal monotone.

Theorem 4 If the source satisfy

$$
\mathbf{J} \in \mathcal{C}^{2}\left([0, T], L^{2}(\Omega)^{3}\right) \quad\left(\text { and } \rho \in \mathcal{C}^{1}\left([0, T], L^{2}(\Omega)\right)\right),
$$


and if the initial data verify

$$
\left(\mathbf{E}_{0}, \mathbf{B}_{0}\right) \in X_{0} \times H_{0}(\operatorname{div} 0, \Omega), \quad \mathbf{E}_{1} \in X_{0}, \mathcal{A} \mathbf{E}_{0}+\mathcal{B} \mathbf{E}_{1} \in L^{2}(\Omega)^{3},
$$

then problem (P1) has a unique solution verifying

$$
\begin{aligned}
& \mathbf{E} \in \mathcal{C}^{1}\left([0, T], X_{0}\right) \cap \mathcal{C}^{2}\left([0, T], L^{2}(\Omega)^{3}\right), \\
& \mathbf{B} \in \mathcal{C}^{2}\left([0, T], H_{0}(\operatorname{div} 0, \Omega)\right), \\
& p \in \mathcal{C}^{1}\left([0, T], L^{2}(\Omega)\right)
\end{aligned}
$$

Proof. According to Lemma 4, we can apply the Hille-Yosida Theorem to the equivalent problem (18). The hypotheses on the source terms and on the data mean that $u_{0} \in D(A)$ and $f \in \mathcal{C}^{1}([0, T], H)$. Then problem (18) has a unique solution $u$ verifying

$$
u \in \mathcal{C}^{0}([0, T], D(A)) \cap \mathcal{C}^{1}([0, T], H) .
$$

The regularity results on $\mathbf{E}, p$ and $\mathbf{B}$ follow readily.

\section{Well-posedness for the hyperbolic correction}

Problem (H1) consists in finding $\mathbf{E}, \mathbf{B}: \Omega \times[0, T] \rightarrow \mathbb{R}^{3}$ and $p: \Omega \times[0, T] \rightarrow \mathbb{R}$ such that

$$
\left\{\begin{array}{l}
\frac{\partial \mathbf{E}}{\partial t}-c^{2} \operatorname{curl} \mathbf{B}+c^{2} \nabla p=-\frac{1}{\epsilon_{0}} \mathbf{J} \\
\frac{\partial \mathbf{B}}{\partial t}+\operatorname{curl} \mathbf{E}=0 \\
\frac{\partial p}{\partial t}+\operatorname{div} \mathbf{E}=\frac{\rho}{\epsilon_{0}}
\end{array}\right.
$$

along with the initial conditions (5) and the boundary conditions

$$
\mathbf{E} \times \mathbf{n}=0 \text { on } \Gamma, \frac{\partial p}{\partial t}+c \frac{\partial p}{\partial \mathbf{n}}=0 \text { on } \Gamma .
$$

Then if $p$ is a sufficiently smooth solution, it satisfies the following system

$$
\left\{\begin{array}{l}
\frac{\partial^{2} p}{\partial t^{2}}-c^{2} \Delta p=\frac{1}{\epsilon_{0}}\left(\frac{\partial \rho}{\partial t}+\operatorname{div} \mathbf{J}\right) \text { on } \Omega \times[0, T] \\
\frac{\partial p}{\partial t}+c \frac{\partial p}{\partial \mathbf{n}}=0 \text { on } \Gamma \times[0, T] \\
p(., 0)=0, \frac{\partial p}{\partial t}(., 0)=0 \text { on } \Omega
\end{array}\right.
$$

$\mathrm{RR} \mathrm{n}^{\circ} 5850$ 
Proposition 4 The variational formulation of problem (20) is: Find $p(t) \in H^{1}(\Omega)$ such that for all $q \in H^{1}(\Omega)$ we have

$$
\frac{d^{2}}{d t^{2}}(p, q)_{0}+c^{2}(\nabla p, \nabla q)_{0}+c \frac{d}{d t} \int_{\Gamma} p q d \Gamma=\frac{1}{\epsilon_{0}}\left(\frac{\partial \rho}{\partial t}+\operatorname{div} \mathbf{J}, q\right)_{0} .
$$

We set, for $p, q \in H^{1}(\Omega)$,

$$
\langle\mathcal{A} p, q\rangle=c^{2}(\nabla p, \nabla q)_{0}, \quad\langle\mathcal{C} p, q\rangle=c \int_{\Gamma} p q d \Gamma .
$$

We thus define bounded linear operators $\mathcal{A}, \mathcal{C} \in \mathcal{L}\left(H^{1}(\Omega), H^{1}(\Omega)^{\prime}\right)$. The variational problem (21) can be rewritten: Find $p(t) \in H^{1}(\Omega)$ such that

$$
\frac{\partial^{2}}{\partial t^{2}} p+\mathcal{A} p+\mathcal{C} \frac{\partial p}{\partial t}=\frac{1}{\epsilon_{0}}\left(\frac{\partial \rho}{\partial t}+\operatorname{div} \mathbf{J}\right) \text { on } H^{1}(\Omega)^{\prime} .
$$

This is finally equivalent to finding $u=\left(p, \partial_{t} p\right) \in H$ such that

$$
\frac{d}{d t} u+A u=f
$$

where we set

$$
\begin{aligned}
A=\left(\begin{array}{cc}
0 & -I \\
\mathcal{A} & \mathcal{C}
\end{array}\right), \quad f=\left(\begin{array}{c}
0 \\
\left(\partial_{t} \rho+\operatorname{div} \mathbf{J}\right) / \epsilon_{0}
\end{array}\right), \\
H=H^{1}(\Omega) \times L^{2}(\Omega), \\
D(A)=\left\{v \in H^{1}(\Omega) \times H^{1}(\Omega) \mid \mathcal{A} v_{1}+\mathcal{C} v_{2} \in L^{2}(\Omega)\right\} .
\end{aligned}
$$

Lemma 5 The operator $I+A: D(A) \rightarrow H$ is maximal monotone.

Proof. We consider the scaled scalar product on $H^{1}(\Omega)$ :

$$
(u, v)_{1, \star}=\int_{\Omega}\left(c^{2} \nabla u \cdot \nabla v+u v\right) d x \quad \text { for } u, v \in H^{1}(\Omega) .
$$

Let $u=\left(u_{1}, u_{2}\right) \in D(A)$.

$$
\begin{aligned}
((I+A) u, u)_{H} & =\int_{\Omega}\left(c^{2}\left|\nabla u_{1}\right|^{2}+u_{1}^{2}+u_{2}^{2}\right) d x+\left(-u_{2}, u_{1}\right)_{1, \star}+\left(\mathcal{A} u_{1}+\mathcal{C} u_{2}, u_{2}\right)_{0} d x \\
& =\int_{\Omega}\left(c^{2}\left|\nabla u_{1}\right|^{2}+u_{1}^{2}+u_{2}^{2}-u_{2} u_{1}\right) d x+c \int_{\Gamma} u_{2}^{2} d \Gamma \\
& \geq 0 .
\end{aligned}
$$


Hence $I+A$ is monotone. Let $f \in H$. We look for $u \in D(A)$ such that $(2 I+A) u=f$, that is

$$
\left\{\begin{array}{l}
2 u_{1}-u_{2}=f_{1} \in H^{1}(\Omega) \\
2 u_{2}+\mathcal{A} u_{1}+\mathcal{C} u_{2}=f_{2} \in L^{2}(\Omega)
\end{array}\right.
$$

which is equivalent to

$$
\left\{\begin{array}{l}
u_{1}=\frac{1}{2}\left(u_{2}+f_{1}\right) \\
4 u_{2}+\mathcal{A} u_{2}+2 \mathcal{C} u_{2}=2 f_{2}-\mathcal{A} f_{1} \in H^{1}(\Omega)^{\prime}
\end{array}\right.
$$

The bilinear form defined from the operator $4 u_{2}+\mathcal{A} u_{2}+2 \mathcal{C} u_{2} \in H^{1}(\Omega)^{\prime}$ is clearly continuous and coercive on $H^{1}(\Omega)^{2}$. The linear form $2 f_{2}-\mathcal{A} f_{1}$ is continuous on $H^{1}(\Omega)$. Applying the Lax-Milgram Theorem, there exists a unique $u_{2} \in H^{1}(\Omega)$ such that $4 u_{2}+\mathcal{A} u_{2}+2 \mathcal{C} u_{2}=$ $2 f_{2}-\mathcal{A} f_{1}$.

Let $u_{1}=\frac{1}{2}\left(u_{2}+f_{1}\right)$, then $u_{1} \in H^{1}(\Omega)$ and $\mathcal{A} u_{1}+\mathcal{C} u_{2}=f_{2}-2 u_{2} \in L^{2}(\Omega)$, hence $u \in D(A)$. The operator $2 I+A: D(A) \rightarrow H$ is onto, hence $I+A$ is maximal monotone.

Define the functional set

$$
E\left(\Delta, H^{1}(\Omega)\right)=\left\{q \in H^{1}(\Omega) \mid \Delta q \in L^{2}(\Omega)\right\} .
$$

Proposition 5 If $p(., 0)=0, \partial_{t} p(., 0)=0$ and $\left(\partial_{t} \rho+\operatorname{div} \mathbf{J}\right) \in \mathcal{C}^{1}\left([0, T], L^{2}(\Omega)\right)$, then problem (21) admits a unique solution $p$ such that

$$
\left\{\begin{array}{l}
p \in \mathcal{C}^{0}\left([0, T], E\left(\Delta, H^{1}(\Omega)\right)\right) \cap \mathcal{C}^{1}\left([0, T], H^{1}(\Omega)\right) \cap \mathcal{C}^{2}\left([0, T], L^{2}(\Omega)\right), \\
\hat{E} \text { and } c \frac{\partial p}{\partial n}+\frac{\partial p}{\partial t}=0 \text { on } \Gamma .
\end{array}\right.
$$

Proof. Applying the Hille-Yosida Theorem, the problem has a unique weak solution

$$
p \in \mathcal{C}^{1}\left([0, T], H^{1}(\Omega)\right) \cap \mathcal{C}^{2}\left([0, T], L^{2}(\Omega)\right) .
$$

For any test function $\varphi \in \mathcal{D}(\Omega)$, the variational formulation reads

$$
\left(\frac{\partial^{2} p}{\partial t^{2}}, \varphi\right)_{0}+c^{2}(\nabla p, \nabla \varphi)_{0}=\frac{1}{\epsilon_{0}}\left(\frac{\partial \rho}{\partial t}+\operatorname{div} \mathbf{J}, \varphi\right)_{0},
$$

whence

$$
\frac{\partial^{2} p}{\partial t^{2}}-\Delta p=\frac{1}{\epsilon_{0}}\left(\frac{\partial \rho}{\partial t}+\operatorname{div} \mathbf{J}\right)
$$

and so $\Delta p \in \mathcal{C}^{0}\left([0, T], L^{2}(\Omega)\right)$. In other words, $p \in \mathcal{C}^{0}\left([0, T], E\left(\Delta, H^{1}(\Omega)\right)\right)$. In particular, there holds, according to the above, $\left.\partial_{t} p\right|_{\Gamma}$ in $H^{1 / 2}(\Gamma)$, and $\left.\partial_{n} p\right|_{\Gamma}$ in $H^{-1 / 2}(\Gamma)$.

For any test function $\varphi \in \mathcal{C}^{\infty}(\bar{\Omega})$, the variational formulation yields

$$
\left(\frac{\partial^{2} p}{\partial^{2} t}-c^{2} \Delta p, \varphi\right)_{0}+c\left\langle c \frac{\partial p}{\partial \mathbf{n}}+\frac{\partial p}{\partial t}, \varphi\right\rangle_{H^{1 / 2}(\Gamma)}=\frac{1}{\epsilon_{0}}\left(\frac{\partial \rho}{\partial t}+\operatorname{div} \mathbf{J}, \varphi\right)_{0} .
$$

$\mathrm{RR} \mathrm{n}^{\circ} 5850$ 
According to equality (23) which is valid in $L^{2}(\Omega)$, we have

$$
\left\langle c \frac{\partial p}{\partial \mathbf{n}}+\frac{\partial p}{\partial t}, \varphi\right\rangle_{H^{1 / 2}(\Gamma)}=0, \forall \varphi \in \mathcal{C}^{\infty}(\bar{\Omega}) .
$$

In other words,

$$
c \frac{\partial p}{\partial \mathbf{n}}+\frac{\partial p}{\partial t}=0 \text { in } H^{-1 / 2}(\Gamma)
$$

Since $\partial_{t} p_{\mid \Gamma}$ belongs to $\mathcal{C}^{0}\left([0, T], H^{1 / 2}(\Gamma)\right)$, the above equality holds in the classical sense.

This result can be improved, when the domain $\Omega$ is convex.

Corollary 1 Assume the domain $\Omega$ is convex, then

$$
p \in \mathcal{C}^{0}\left([0, T], H^{2}(\Omega)\right) .
$$

Proof. This is a straightforward consequence of the fact that, since $\partial_{n} p_{\mid \Gamma}$ belongs to $\mathcal{C}^{0}\left([0, T], H^{1 / 2}(\Gamma)\right), \nabla p$ is a continuous function of $t$ in the space

$$
\left\{\mathbf{f} \in H(\operatorname{curl}, \Omega) \cap H(\operatorname{div}, \Omega) \mid \mathbf{f} \cdot \mathbf{n}_{\mid \Gamma} \in H^{1 / 2}(\Gamma)\right\} .
$$

According to [7][Chapter IX, pp. 247-248], this functional space is actually imbedded in $H^{1}(\Omega)^{3}$ when $\Omega$ is convex.

We then conclude by solving the hyperbolically corrected problem (H1).

Corollary 2 Assume that the sources verify

$$
\rho \in \mathcal{C}^{2}\left([0, T], L^{2}(\Omega)\right), \quad \mathbf{J} \in \mathcal{C}^{1}([0, T], H(\operatorname{div}, \Omega)),
$$

and that the initial conditions verify

$$
p(., 0)=0, \frac{\partial p}{\partial t}(., 0)=0, \quad\left(\mathbf{E}_{0}, \mathbf{B}_{0}\right) \in X_{0} \times X .
$$

Then problem (H1) has a unique solution with $p$ as in (22) and

$$
\mathbf{E} \in \mathcal{C}^{0}\left([0, T], X_{0}\right) \cap \mathcal{C}^{1}([0, T], H(\operatorname{div}, \Omega)), \quad \mathbf{B} \in \mathcal{C}^{0}([0, T], X) \cap \mathcal{C}^{1}\left([0, T], H_{0}(\operatorname{div}, \Omega)\right) .
$$

Proof. As $\left(\partial_{t} \rho+\operatorname{div} \mathbf{J}\right) \in \mathcal{C}^{1}\left([0, T], L^{2}(\Omega)\right)$, we can apply the previous Proposition whence the existence and uniqueness of

$$
p \in \mathcal{C}^{0}\left([0, T], E\left(\Delta, H^{1}(\Omega)\right)\right) \cap \mathcal{C}^{1}\left([0, T], H^{1}(\Omega)\right) \cap \mathcal{C}^{2}\left([0, T], L^{2}(\Omega)\right),
$$

solution to (20). This implies in turn

$$
\left(\frac{\mathbf{J}}{\epsilon_{0}}+c^{2} \nabla p\right) \in \mathcal{C}^{0}([0, T], H(\operatorname{div}, \Omega)), \hat{E} \text { and }\left(\frac{\rho}{\epsilon_{0}}-\frac{\partial p}{\partial t}\right) \in \mathcal{C}^{1}\left([0, T], L^{2}(\Omega)\right) .
$$

INRIA 
Moreover, $p$ being solution to (20), we have

$$
\frac{\partial}{\partial t}\left(\frac{\rho}{\epsilon_{0}}-\frac{\partial p}{\partial t}\right)+\operatorname{div}\left(\frac{\mathbf{J}}{\epsilon_{0}}+c^{2} \nabla p\right)=\frac{1}{\epsilon_{0}}\left(\frac{\partial \rho}{\partial t}+\operatorname{div} \mathbf{J}\right)-\frac{\partial^{2} p}{\partial t^{2}}+c^{2} \Delta p=0 .
$$

As expected, $p$ accounts for the targeted hyperbolic correction on the continuity equation (1). We can then apply Theorem 1 to conclude. Indeed, use the classical setting

$$
u=\left(\begin{array}{c}
\mathbf{E} \\
c \mathbf{B}
\end{array}\right), A=\left(\begin{array}{cc}
0 & -c \operatorname{curl} \\
c \operatorname{curl} & 0
\end{array}\right), f=\left(\begin{array}{c}
\mathbf{J} / \epsilon_{0}+c^{2} \nabla p \\
0
\end{array}\right), u_{0}=\left(\begin{array}{c}
\mathbf{E}_{0} \\
c \mathbf{B}_{0}
\end{array}\right)
$$

with $H=L^{2}(\Omega)^{3} \times L^{2}(\Omega)^{3}$, and $D(A)=H_{0}(\operatorname{curl}, \Omega) \times H(\operatorname{curl}, \Omega)$. The assumptions of Theorem 1 are easily checked. The additional regularity on the divergence of the electromagnetic field, and the vanishing normal trace of the magnetic field, stem from the relationships

$$
\operatorname{div} \mathbf{E}=\frac{\rho}{\epsilon_{0}}-\frac{\partial p}{\partial t}, \text { and } \frac{\partial \mathbf{B}}{\partial t}=-\operatorname{curl} \mathbf{E}
$$

\section{References}

[1] F. Assous, P. Ciarlet, Jr., P.-A. Raviart, E. Sonnendrücker, A characterization of the singular part of the solution to Maxwell's equations in a polyhedral domain, Math. Meth. Appl. Sci. 22 (1999) pp. 485-499.

[2] F. Assous, P. Degond, E. Heintze, P. A. Raviart, J. Segré, On a finite element method for solving the three-dimensional Maxwell equations, Journal of Computational Physics 109 (1993) pp. 222-237.

[3] C. K. Birdsall, A. B. Langdon, Plasma physics via computer simulation, Institute of Physics, Bristol (1991) p. 359.

[4] P. Blaise, P. Ciarlet, Jr., R. Sentis, Développements récents dans le code de résolution des équations de Vlasov-Maxwell en 2D structuré MANET, Technical Report CEA/DRIF/DCSA/MLS/B3 DO 224/97, 91680 Bruyères-le-Châtel, France (In French).

[5] J. P. Boris, Relativistic plasma simulations - Optimization of a hybrid code, Proc. 4th Conf. Num. Sim. of Plasmas (NRL Washington, Washington DC, 1970) pp. 3-67.

[6] H. Brézis, Analyse fonctionnelle, Dunod, Paris (1999).

[7] R. Dautray, J. L. Lions, Analyse mathématique et calcul numérique pour les sciences et les techniques, Masson, Paris (1988). 
[8] P. Fernandes, G. Gilardi, Magnetostatic and electrostatic problems in inhomogeneous anisotropic media with irregular boundary and mixed boundary conditions, Math. Models Methods Appl. Sci. 7 (1997) pp. 957-991.

[9] V. Girault, P. A. Raviart, Finite Element Methods for Navier-Stokes Equations, Springer Verlag, Berlin (1986).

[10] A. B. Langdon, On enforcing Gauss' law in electromagnetic particle-in-cell codes, Computer Physics Communisations 70 (1992) pp. 447-450.

[11] B. Marder, A method for incorporating Gauss's law into electromagnetic PIC codes, J. Comput. Phys. 68 (1987) pp. 48-55.

[12] C.D. Munz, R. Schneider, E. Sonnendrücker, U. Voss, Maxwell's equations when the charge conservation is not satisfied, C. R. Acad. Sci. Paris, Ser. I 328 (1999) pp. 431436.

[13] C.-D. Munz, P. Omnes, R. Schneider, E. Sonnendrücker, U. Voss (2000) : Divergence correction techniques for Maxwell solvers based on a hyperbolic model, J. Comput. Phys. 161, no. 2, pp. 484-511.

[14] J. Villasenor, O. Buneman, Rigorous charge conservation for local electromagnetic field solvers, Comput. Phys. Commun. 69 (1992) pp. 306-316. 


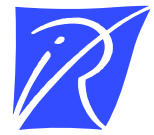

Unité de recherche INRIA Lorraine

LORIA, Technopôle de Nancy-Brabois - Campus scientifique 615, rue du Jardin Botanique - BP 101 - 54602 Villers-lès-Nancy Cedex (France)

Unité de recherche INRIA Futurs : Parc Club Orsay Université - ZAC des Vignes

4, rue Jacques Monod - 91893 ORSAY Cedex (France)

Unité de recherche INRIA Rennes : IRISA, Campus universitaire de Beaulieu - 35042 Rennes Cedex (France)

Unité de recherche INRIA Rhône-Alpes : 655, avenue de l'Europe - 38334 Montbonnot Saint-Ismier (France) Unité de recherche INRIA Rocquencourt : Domaine de Voluceau - Rocquencourt - BP 105 - 78153 Le Chesnay Cedex (France) Unité de recherche INRIA Sophia Antipolis : 2004, route des Lucioles - BP 93 - 06902 Sophia Antipolis Cedex (France) 